\title{
Short-Term Wind Power Forecasting Based on Spatiotemporal Correlations
}

\author{
Jinli Dou ${ }^{1, a}$, Chun Liü, b and Bo Wang ${ }^{\mathrm{c}}$ \\ ${ }^{1}$ State Key Laboratory of Operation and Control of Renewable Energy \& Storage Systems, China. \\ ${ }^{2}$ China Electric Power Research Institute, Beijing, China, 100192, China. \\ avictordou@126.com, bliuchun@epri.sgcc.com.cn, ‘wangbo@epri.sgcc.com.cn
}

\begin{abstract}
Keywords: Short-Term wind power forecasting, Deep Neural Network, Convolutional Neural Network, spatiotemporal correlations.
\end{abstract}

\begin{abstract}
Wind power forecasting is of great significance for promoting the new energy resources accommodation and the economic efficiency, security and stability of power grid operation. The traditional wind power forecasting model has monotonous elements for input and demonstrates a lack of spatiotemporal correlation between elements. Therefore, a deep neural network model considering space-time correlation is proposed. Firstly, a multi-dimensional space-time data input modeling method is proposed based on meshed numerical weather forecasting. Then, a variety of deep neural network models for wind power prediction are established. Multi-layer CNN (Convolutional Neural Networks) are utilized for feature extraction, meanwhile LSTM (Long Short-Term Memory) networks are used for pattern memory. Finally, the prediction of single station and regional wind power is carried out respectively, which proves the effectiveness and feasibility of the method. The results show that, compared with the traditional single-layer neural network, the deep neural network in this paper can effectively mine the spatiotemporal correlation between data and improve the prediction accuracy of single-station wind power forecasting.
\end{abstract}

\section{Introduction}

Wind energy is the fastest growing renewable energy source around the world. During the 12th FYP period, China's wind power generation installed capacity ranked the first in the world for five consecutive years, with a cumulative increase of 98 million kilowatts. In 2016, the newly-added wind power installed capacity was 19.3 million kilowatts, Cumulative installed capacity of the grid reached 149 million kilowatts, accounting for $9 \%$ of the total installed capacity of power generation, becoming the third largest source of energy after China's thermal power and hydropower [1]. The curtailment of wind power has become a major factor that restricts the development of wind power. Firstly, wind power itself is characterized by volatility, randomness and intermittency. On the other hand, wind power development is so fast that the power grid construction cannot keep up.

Wind power forecasting is an important reference for wind farm site selection as well as large-scale grid connection and operation. Increasing the wind power forecasting accuracy guarantees the economic dispatch and power balance of the power system and can also reduce the configuration of energy storage capacity cost. Due to the good application of machine learning in nonlinear systems, algorithms of machine learning such as artificial neural network, support vector machine, and maximum entropy algorithm are widely used in prediction algorithms. In addition, there are also applications of autoregressive moving average method, Kalman filter method, wavelet analysis and a series of data analysis methods [2]. Literature [3] inputs the elements related to wind power, such as wind speed, wind direction, temperature, pressure and humidity, to the artificial neural network, and establishes the basic model of wind power forecasting. Since then, many researches on wind power prediction accuracy have focused on the following aspects: In data preprocessing, literature [4] combined the data of several different NWP modes by dynamic correction of weighting coefficients to improve the prediction accuracy. The accuracy of short-term wind power forecasting is improved by classifying and correcting NWP errors by combining K-means clustering method and 
artificial neural network [5]. In the literature [6], deep neural network model is used to predict the wind power with higher accuracy whose input of the model is the power and NWP wind speed.

The algorithms used in the above wind power predictions are mostly designed based on single-layer neural network. The disadvantage of the algorithm is that the input elements are limited and the spatial-temporal correlation between the weather elements such as NWP cannot be fully mined. On the other hand, as the number of input elements increases, the training difficulty increases simultaneously and the generalization ability decreases due to the increase of redundant information, which leads to the increase of prediction error. Based on the above limitations, this paper proposes an input model based on multiple temporal and spatial elements to provide the foundation for data mining. On the other hand, a deep neural network model is established to effectively mine the correlation among the various elements. Finally, the model is verified and analyzed by the example that model can effectively improve single-station power prediction accuracy with comparison to single-layer neural network.

\section{Input Models Considering the Spatial-Temporal Correlation}

Combining the advantages and disadvantages of multiple wind power prediction algorithms inputs, the forecast input mode adopted in this paper is shown in Fig. 1, and the NWP elements of a single time section are arranged together in time to form a two-dimensional input. The horizontal axis of the input is the time near the point is predicted while the vertical axis of input is the NWP element that affects the wind power output. For example, taking $4 \mathrm{~h}$ near the predicted time point as the time window of whose resolution is $15 \mathrm{~min}$, corresponding to the time point of 17 ; take the NWP wind speed / wind direction sine / wind direction cosine / temperature / humidity / pressure elements as input, there are $8 \times 17=136$ input elements that can be constructed as a set of two-dimensional data. The two sets of data are arranged laterally in a time-dependent manner, and vertically related elements (for example, a wind speed of 100 meters and a wind speed of 30 meters) are arranged together.

The main advantage of this input mode is that it integrates the diversity and time correlation of wind power input elements and constructs a certain two-dimensional structure. Theoretically, the accuracy of wind power prediction can be further improved by mining the temporal correlation of multiple elements.

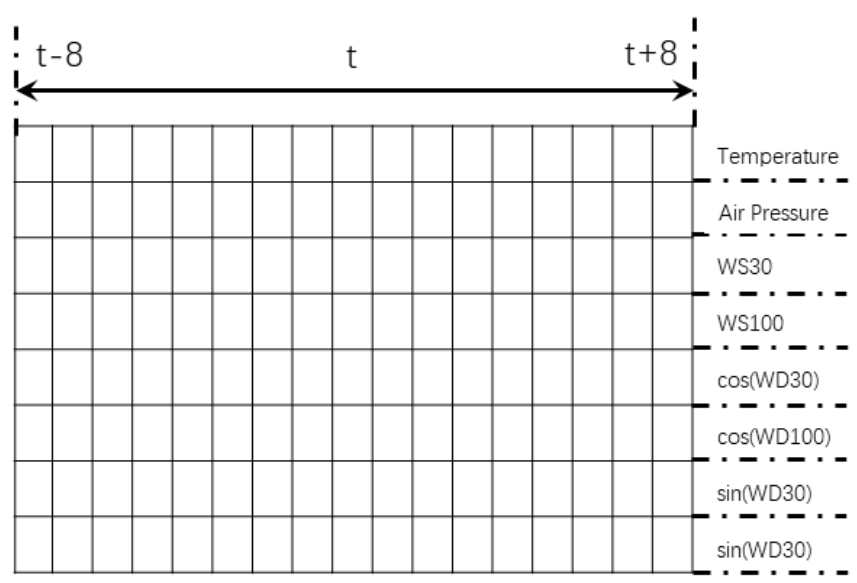

Fig 1. NWP elements / time two-dimensional input model

\section{Wind Power Forecasting Models Based on Deep Neural Network}

\subsection{Convolutional Neural Network}

Convolutional neural network is the first successfully applied deep neural network, LeCun handwritten digital recognition network designed in 1998, with 3 convolutional layers, 2 Pooling layers, 2 fully connected layers is one of most classic convolutional neural networks [14]. Nowadays, 
convolutional neural network has been widely used in computer vision. Using convolutional neural network can effectively solve the problem of image and video recognition and classification. Convolutional neural network has the following two characteristics:

(1) Local connection, in the process of convolution operation, each convolution kernel relates to the local receptive field to extract the parameters in the receptive field and calculate it as a parameter according to the given formula, which can avoid the explosive growth in the amount of training parameters while the inputs parameters are excessive.

(2) Parameter sharing, convolution operation of multiple convolution kernel parameters is the same, with the local connection, the training parameters local can be apparently reduced with esurience of the network's feature extraction capabilities.

\subsection{LSTM Network}

The basic recurrent neural network is a kind of neural network trained by BPTT (Back Propagation Through Time). It has a unique advantage in dealing with time series data, but it has the problem of gradient disappearance on a long-time scale and does not have the memory function on a long-time scale. Correspondingly, the LSTM network has a non-linear structure that can perform long-term memory and has been successfully applied in large-scale text recognition and machine translations.

\subsection{Wind Power Forecasting Model}

As shown in the following Fig.4, the model has three convolutional network layers with multiple lays followed. The convolutional neural network is used to extract the spatiotemporal correlation feature. The pooling layer can reduce the training parameters. The dropout layer is a pattern memory that can reduce the overfitting while the LSTM network layer is used for pattern memory.

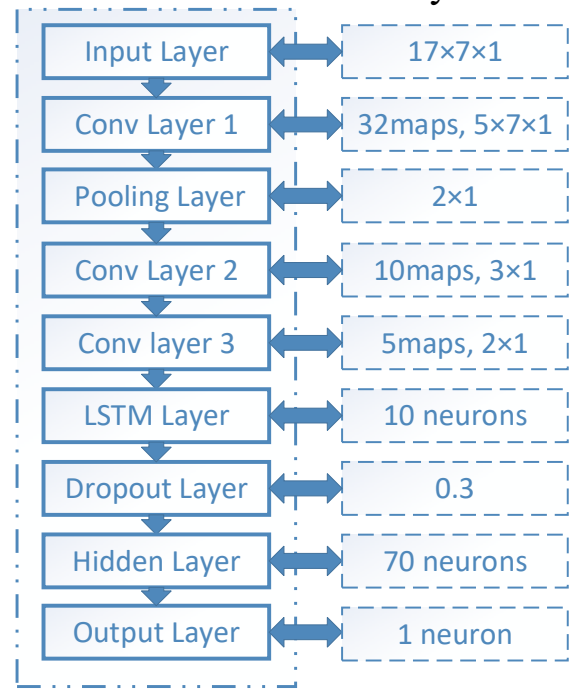

Fig 2. DNN for wind power forecasting

\section{Example Verification}

The evaluation criteria of the wind power forecasting errors are mainly included as follows:

Root mean square error:

$$
E_{r m s}=\frac{\sqrt{\sum_{i=1}^{n}\left(P_{M i}-P_{P i}\right)^{2}}}{S_{o p} \cdot \sqrt{n}}, i=1, \cdots, n
$$

Mean absolute error:

$$
E_{m a}=\frac{1}{n} \sum_{i=1}^{n}\left(\frac{\left|P_{M i}-P_{P i}\right|}{S_{o p}}\right), i=1, \cdots, n
$$

A total of 65,166 sets of data were collected from a wind farm in Shandong Province from November 2013 to March 2016. Among them, the data of the first 45,000 sets were for training and 
the data of 20166 set were utilized as test data. The actual power, the forecast result of single-layer feedforward neural network and the depth Neural network prediction results as shown in Fig.3:

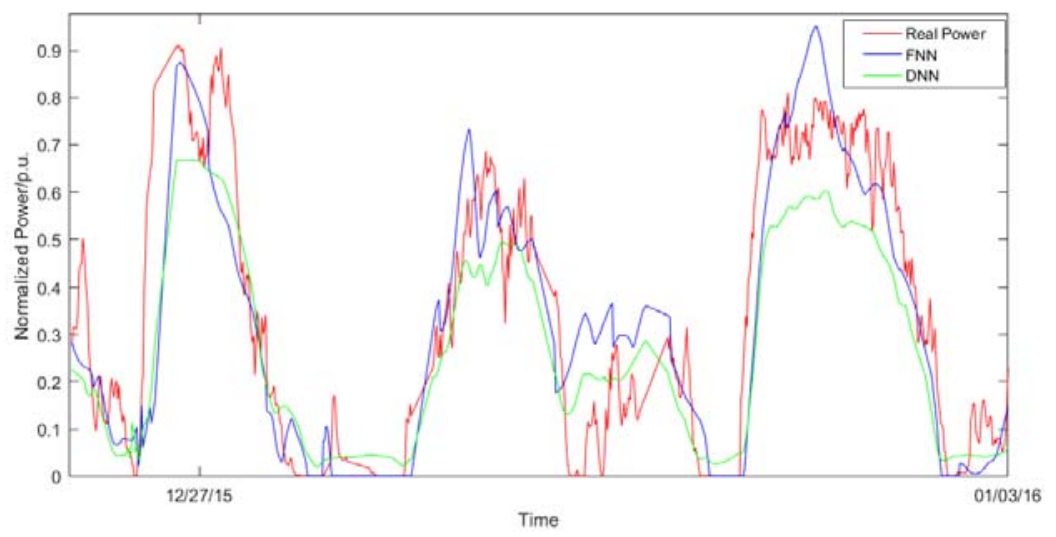

Fig 3. Forecasting Results Comparison

The application of various types of deep neural network prediction error evaluation parameters are shown in Table 1:

Table 1. Error Evaluation Parameters of Different networks

\begin{tabular}{cccccc}
\hline & $3 *$ CNN+LSTM & FNN & CNN & LSTM & CNN+LSTM \\
\hline$E_{\text {rms }}$ & $13.37 \%$ & $15.81 \%$ & $14.15 \%$ & $13.85 \%$ & $13.47 \%$ \\
$E_{\text {та }}$ & $9.16 \%$ & $10.71 \%$ & $9.12 \%$ & $9.40 \%$ & $9.51 \%$ \\
\hline
\end{tabular}

\section{Summary}

It can be concluded that the deep neural network with multi-convolutional layers and LSTM layer has the best prediction effect, the root mean square error is reduced by $2 \%$, and the mean absolute error is reduced by nearly $1.5 \%$. However, the convolutional neural network has a limited decrease in the model error of multi-NWP data input, and the mining of the spatial correlation among data needs to be strengthened.

\section{Acknowledgements}

This article is supported by Key Technology Project of State Grid Corporation of China (NY71-16-046).

\section{References}

[1]. Electric Power Planning \&Electric Institute. Annual Report on China's Energy Development (2016). Beijing: EPPEI, 2016.

[2]. Gu Xingkai, Fan Gaofeng, Wang Xiaorong, et al. Summarization of wind power prediction technology[J]. Power System Technology, 2007, 31(S2): 335-338(in Chinese).

[3]. Fan Gaofeng, Wang Weisheng, Liu Chun, et al. Wind power prediction based on artificial neural network[J]. Proceedings of the CSEE, 2008, 28(34): 118-123(in Chinese).

[4]. Wang Zheng, Rui Pestana, Feng Shuanglei, et al. Short-Term Wind Power Combination Forecasting Method Based on Dynamic Coefficient Updating [J]. Power System Technology, 2017, 41(02): 500-507(in Chinese).

[5]. Xu Qianyao, He Dawei, Zhang Ning. A Short-Term Wind Power Forecasting Approach with Adjustment of Numerical Weather Prediction Input by Data Mining. IEEE Transactions on Sustainable Energy, 2015, 6(4): 1283-1291. 